Proceedings

\title{
The clinical potential of point-of-care quantitative SpectroChip coupled with lateral flow immunoassay in COVID-19 pan- demic
}

Pang-Yen Chen ${ }^{1}$, Cheng-Hao Ko ${ }^{2}$, C.Jason Wang ${ }^{3}$, I-Jen Wang 4,*

1. Department of Emergency Medicine, Mackay Memorial Hospital, Taipei, Taiwan Institute of Environmental and Occupational Health Sciences, School of Medicine, National Yang-Ming University, Taipei, Taiwan 2. SpectroChip Inc., Hsinchu, Taiwan Graduate Institute of Automation and Control, National Taiwan University of Science and Technology, Taipei, Taiwan

3. Department of Pediatrics and Medicine, Stanford University School of Medicine, Stanford, United States

4. Department of Pediatrics, Taipei Hospital, Ministry of Health and Welfare, Taipei, Taiwan

* Correspondence: wij636@gmail.com

\begin{abstract}
Coronavirus disease (COVID-19) is the current grand global public health challenge. To assess the body's immune response from natural COVID-19 disease or from the effects of vaccines, it is necessary to have a method that meets the rapid quantitative detection of antibodies. We integrated a newly designed spectrochip to our COVID-19 test strip procedures to provide sensitive, quantitative capacity to a lateral flow immunoassay (LFIA). Optical interpretation of results by quantitative $\alpha$ index was taken, rather than visual qualification. This detection method can quickly produce results in 6 minutes. We compared the developed product with several other serological IgM/IgG antibody reagents on the market by recruiting 111 participants of suspected COVID-19 infection from March to May 2020 in a single hospital. Taking RT-PCR positive individuals as the gold standard, the analytical platform (Spectrochip +ACE Biolab) can correctly detect all 12 COVID19 patients (100\% sensitivity, 12/12). In contrast, the sensitivity for ACE Biolab alone is $91.67 \%$ $(11 / 12)$, for Biomedomics is $58.33 \%$ (7/12). Methods that use Nucleocapsid (N) + Spike (S) solid-phase antigen (i.e., ACE Biolab, TBG, and Spectrochip +ACE Biolab) perform better, compared with those that use Nucleocapsid (Biomedomics) or Spike (ASK) alone. This new platform's extraordinary detection ability demonstrated clinical potential.
\end{abstract}

Keywords: COVID-19; Lateral flow immunoassay; RT-PCR; Serological test; Quantitative immunoassay

Publisher's Note: MDPI stays neutral with regard to jurisdictional claims in published maps and institutional affiliations.

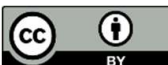

Copyright: (c) 2021 by the authors. Submitted for possible open access publication under the terms and conditions of the Creative Commons Attribution (CC BY) license (http://creativecommons.org/licenses/by/4.0/). 